\title{
$\begin{array}{ll}\text { Research Square } & \text { Preprints are preliminary reports that have not undergone peer review. } \\ \text { They should not be considered conclusive, used to inform clinical practice, }\end{array}$ or referenced by the media as validated information. \\ Lentivirus-Mediated Small Interfering RNA Targeting Piezo1 Inhibits Cartilage Matrix degeneration in Osteoarthritis Chondrocytes
}

\section{Yikai Liu}

the Affiliated Hospital of Qingdao University

Xinzhe Lu

the Affiliated Hospital of Qingdao University

\section{Zian Zhang}

the Affiliated Hospital of Qingdao University

\section{Tao Li}

the Affiliated Hospital of Qingdao University

Haining Zhang ( $\square$ zhanghaining1976@126.com )

The Affiliated Hospital of Qingdao University

\section{Research Article}

Keywords: Chondrocytes, Cartilage matrix, Osteoarthritis, Mechanical stress, Piezo1, Small Interfering RNA

Posted Date: February 16th, 2022

DOI: https://doi.org/10.21203/rs.3.rs-1262556/v1

License: (a) (i) This work is licensed under a Creative Commons Attribution 4.0 International License. Read Full License 


\section{Abstract}

Osteoarthritis $(\mathrm{OA})$ is triggered by abnormal distraction stress, which eventually leads to cartilage degeneration. However, it remains unclear how the mechanical stress factor acts an effect on the destruction of the cartilage matrix. As a kind of mechanical sensitive ion channel protein, Piezo1 has the ability to sense and transmit mechanical signals to cells. Previous studies have found that the stress sensitivity of Piezo1 is closely related to the directionality of mechanical stress and the presence of extracellular matrix. This research aimed to explore the role of lentivirus-mediated Piezo1-siRNA transfection in extracellular matrix in chondrocytes of cultured osteoarthritis in vitro and to investigate the effect of Piezo1 on the progression of osteoarthritis. In this study, we demonstrated that the expression of Piezo1 mRNA in transfection group was significantly decreased after 24h mechanical stress was applied. However, the expression trend of collagen II and aggrecan was opposite to that of Piezo1, matrix metalloproteinase-13 (MMP-13), and a disintegrin and metalloproteinase with thrombospondin motifs 5 (ADAMTS5). Our results indicate that Piezo1 could be used as a possible therapeutic target to attenuate the destruction of cartilage matrix induced by mechanical stress. Lentiviral-mediated Piezo1-siRNA can effectively interfere with the expression of Piezo1 and protect the expression of the extracellular cartilage matrix.

\section{Impact Statement}

Osteoarthritis $(\mathrm{OA})$ is a degenerative disease charactered cartilage abrasion and degeneration, with destruction of extracellular matrix. Abnormal mechanical stress is a key factor in OA pathology. Piezo1 is an ion channel that senses and transmits mechanical signals to cells. To investigate the expression and effect of Piezo1 in chondrocyte, we used Piezo1 inhibition mediated by lentivirus-mediated siRNA on chondrocytes, and chondrocytes underwent mechanical stress to simulate in vivo conditions. Our results showed that inhibition of Piezo1 on chondrocyte reduced MMP13, ADAMTS5 expression and promoted Aggrecan expression, which indicated that Piezo1 could be used as a possible therapeutic target to attenuate the destruction of cartilage matrix induced by mechanical stress. Lentiviral-mediated Piezo1siRNA can effectively interfere with the expression of Piezo1 and protect extracellular cartilage matrix.

\section{Introduction}

Osteoarthritis $(\mathrm{OA})$ is one of the crucial causes of chronic joint pain in the elderly. It can seriously interfere with the patient's daily activities, performance, and may eventually lead to lower extremity disorders and disabilities ${ }^{1,2}$. Women and the elderly are susceptible to OA. The main symptoms include varying degrees of dysfunction and joint pain, and the quality of life of patients is severely affected ${ }^{3}$. OA is triggered by the macroscale stress related with joint kinematics, which is eventually converted into nanoscale stress at the individual extracellular matrix and cytoskeleton level, at which the mechanical failure ultimately result in joint pathology ${ }^{4-7}$. The primary pathology of OA are the decrease of chondrocytes, the degradation of extracellular matrix and the sclerosis of subchondral bone ${ }^{8}$. During the past few decades, OA has been identified as a kind of wear and tear disease, which can result in articular cartilage destruction and 
disability. The prevalent conception is that the increased fragility of the cartilage, joint instability, and increased pressure on weight-bearing joints served as the major predisposing factors ${ }^{9}$. Chondrocytes and cartilage matrix can support force and regulate their own metabolic activities in response to mechanical loading ${ }^{10-12}$. Under abnormal stress conditions, such as trauma and chronic strain, mechanical stress acts a crucial effect on the pathogenesis of osteoarthritis ${ }^{13,14}$.

Cartilage matrix is consists predominantly of collagen and proteoglycans, of which type II collagen (col-2) accounted for $90 \%$ of the total collagen, other types of collagen less; aggrecan is the highest proportion of proteoglycan in cartilage ${ }^{15}$. The most critical role of the cartilage matrix is to resist tensile stress and provide tissue tensile strength and stiffness ${ }^{16}$. The excessive collapse of col- 2 and aggrecan caused by abnormal distraction stress is a critical factor in osteoarthritis cartilage degeneration ${ }^{17}$. However, it remains unclear whether the mechanical stress factor acts an effect on the destruction of the cartilage matrix. The pivotal mediators of cartilage matrix degradation can be classified into two categories, including the closely related a disintegrin and metalloproteinase with thrombospondin motifs (ADAMTS) and matrix metalloproteinases (MMPs), which is a group of zinc-dependent proteases degrading col-2 ${ }^{18-21}$. MMPs covers a variety of branches, such as MMP-1, -8 , and -13 , among which the aggrecan can be degraded by both ADAMTS and MMPs ${ }^{22,23}$. Unlike other MMPs, MMP-13 cleaves not only col-2 but also fibrinogen. In this, research, MMP-13 and ADAMTS5 were detected in order to explore the relationship between extracellular matrix degradation and Piezo1 protein.

Mechanosensitive channels can be used to mediate several coefficients, such as blood pressure regulation, proprioception, hearing, and touch ${ }^{24}$. It was until the discovery of the evolutionarily-conserved Piezo family engaged in mechano-transduction that the molecular mechanism of the mechanical sensitive ion channels was discovered ${ }^{25}$. Correctly, Piezo1 was regarded as molecules engaged in mechano-sensation channels, which were capable of sensing mechanical signals and keeping the cell volume homeostasis ${ }^{26,27}$. After being treated with the specific cationic blocker GsMTx4, it can act a beneficial effect on the chondrocytes of the stress model and reduce the rate of chondrocyte apoptosis induced by traumatic factors ${ }^{28}$. Previous studies have found that the stress sensitivity of Piezo1 is closely related to the directionality of mechanical stress and the presence of extracellular matri ${ }^{29}$. Chondrocytes are mechano-sensation cells, so it is meaningful that Piezo1 could act an essential effect on the degradation of the OA cartilage matrix.

In this study, Piezo1 was used as the target gene to design specific siRNA targeting Piezo1 and transfected into human OA chondrocytes with a lentiviral vector. To study the role of Piezo1 in the extracellular cartilage matrix, it is intended to provide new ideas for gene therapy of OA.

\section{Materials And Methods}

\section{Isolation and culture of OA chondrocytes}

This project was approved by the Ethics Committee of the Affiliated Hospital of Qingdao University. All patients were informed and signed consent. Chondrocytes were harvested from articular cartilage derived 
from the OA patients undergoing total knee arthroplasty. Cartilage tissue were briefly washed with phosphate-buffered saline (PBS) containing $400 \mathrm{U} / \mathrm{ml}$ penicillin and $0.4 \mathrm{mg} / \mathrm{ml}$ Streptomycin (Hyclone, USA) for 3 times. The samples were cut into $1 \mathrm{~mm} 3$ granules under aseptic conditions. Then trypsin and collagenase (Solaibio, China) sequential digestion for $4 \mathrm{~h}$, the cells were collected after $1000 \mathrm{r} / \mathrm{min}$ centrifugation. $\mathrm{OA}$ chondrocytes were cultured in $25 \mathrm{~cm}^{2}$ flask density of $2 \times 10^{4} / \mathrm{cm}^{2}$ which containing medium composed of DMEM (Hyclone, USA) provided with $15 \%$ fetal bovine serum (Hyclone, USA). The cells were cultured under the conditions of $5 \% \mathrm{CO}_{2}$ at $37^{\circ} \mathrm{C}$, and the culture medium was replaced for a period of three days.

\section{Preparation and transfection of small interfering RNA}

The construction of the virus vector was completed by Gemma gene (Shanghai). All sequences were specific oligonucleotide chains (Table 1). Virus titer was determined by hole-by-hole dilution titer assay $(1.0 \times 108 \mathrm{TU} / \mathrm{mL})$ after virus collection. The chondrocytes were divided into 5 groups blank control, Piezo1siRNA1-3 and negative control. Totally $50 \mu$ lentivirus vector, containing each interference sequences and negative control sequences was added to medium, respectively. The titer is $1 \times 10^{8} \mathrm{TU} / \mathrm{ml}$. The same amount of medium solution was added in blank control. After $48 \mathrm{~h}$, the cells were observed and counted under fluorescence microscope. 
Table 1

Interfering sequences and negative control sequences.

\begin{tabular}{|c|c|}
\hline $\begin{array}{l}\text { Oligo } \\
\text { Name }\end{array}$ & Oligo Sequence \\
\hline \multirow[t]{2}{*}{$\begin{array}{l}\text { Piezo1- } \\
\text { siRNA1 }\end{array}$} & $\begin{array}{l}\text { 5'- } \\
\text { GATCCGCGTCATCATCGTGTGTAAGATTCAAGAGATCTTACACACGATGATGACGCTTTTTTG- } \\
3^{\prime}\end{array}$ \\
\hline & $\begin{array}{l}\text { 3'- } \\
\text { AATTCAAAAAAGCGTCATCATCGTGTGTAAGATCTCTTGAATCTTACACACGATGATGACGCG- } \\
5^{\prime}\end{array}$ \\
\hline \multirow[t]{2}{*}{$\begin{array}{l}\text { Piezo1- } \\
\text { siRNA2 }\end{array}$} & $\begin{array}{l}\text { 5'- } \\
\text { GATCCGCGTCTTCCTTAGCCATTACTTTCAAGAGAAGTAATGGCTAAGGAAGACGCTTTTTTG- } \\
3^{\prime}\end{array}$ \\
\hline & $\begin{array}{l}\text { 3'- } \\
\text { AATTCAAAAAAGCGTCTTCCTTAGCCATTACTTCTCTTGAAAGTAATGGCTAAGGAAGACGCG- } \\
5^{\prime}\end{array}$ \\
\hline \multirow[t]{2}{*}{$\begin{array}{l}\text { Piezo1- } \\
\text { siRNA3 }\end{array}$} & $\begin{array}{l}5^{\prime}- \\
\text { GATCCGCCTCAAGTACTTCATCAACTTTCAAGAGAAGTTGATGAAGTACTTGAGGCTTTTTTG- } \\
3^{\prime}\end{array}$ \\
\hline & $\begin{array}{l}3^{\prime}- \\
\text { AATTCAAAAAAGCCTCAAGTACTTCATCAACTTCTCTTGAAAGTTGATGAAGTACTTGAGGCG- } \\
5^{\prime}\end{array}$ \\
\hline \multirow[t]{2}{*}{$\begin{array}{l}\text { Negative } \\
\text { control }\end{array}$} & $\begin{array}{l}\text { 5'- } \\
\text { GATAGCGTCCATCATCGTGTGTAAGATTCAAGAGATCTTACACACGATGATGACGCTTTTTTG- } \\
3^{\prime}\end{array}$ \\
\hline & $\begin{array}{l}\text { 3'- } \\
\text { AATAGCGTCAAGCGTCATCATCGTGTGTAAGATCTCTTGAATCTTACACACGATGATGACGCG- } \\
5^{\prime}\end{array}$ \\
\hline
\end{tabular}

\section{Application of cyclic stress}

The chondrocytes were inoculated into the Flexcell aseptic membranous 6-well plate, cultured in $2.5 \mathrm{ml}$ DMEM containing $15 \%$ fetal bovine serum each well. When cells fusion reaches $40 \%$, added the selected effective lentivirus and the empty vector virus $50 \mu \mathrm{l}$ per well. The virus titer is $1 \times 108 \mathrm{TU} / \mathrm{ml}$. The culture plates were placed in the multichannel cell stress loading system FX-4000T (Flexcell, USA) in $5 \% \mathrm{CO}_{2}$ incubator at $37^{\circ} \mathrm{C}$ in a humid atmosphere. Because of the different stress time applied to cells, each group was divided into five subgroups, and the cyclic stress of $0 \mathrm{~h} / 2 \mathrm{~h} / 12 \mathrm{~h} / 24 \mathrm{~h} / 48 \mathrm{~h}$ was loaded respectively, with the amplitude of $20 \%$ and the period of 10 times / min.

\section{qRT-PCR}

Each group added RNAiso (TaKaRa, Japan) to extract the total RNA. PrimeScript RT reagent Kit (TaKaRa, Japan) was used to reverse RNA to cDNA. Then used SYBR Premix ExTaq®kit (TaKaRa, Japan) for RT-PCR. The CT values of Piezo1, MMP-13, ADAMTS5, and Aggrecan genes were obtained by FTC-2000 system (Applied Biosystems, China) and the parameters were as follows: pre denatured: $95^{\circ} \mathrm{C}$ for 30 seconds, 1 
cycle; PCR: $95^{\circ} \mathrm{C}$ for 5 seconds, $60^{\circ} \mathrm{C}$ for 30 seconds, $72^{\circ} \mathrm{C}$ for 30 seconds for 40 cycles. The relative expression of target gene was calculated by $2^{-\triangle \Delta C t}$. GAPDH, Piezo1, MMP-13, ADAMTS5, and Aggrecan primers were produced by Shanghai Bioengineering Company (Table 2).

Table 2

The oligo sequences of the target genes.

\begin{tabular}{|ll|}
\hline Oligo Name & Oligo Sequence \\
\hline Piezo-1 & $\begin{array}{l}\text { Forward PrimerCATCTTGGTGGTCTCCTCTGTCT } \\
\text { Reverse Primer CTGGCATCCACATCCCTCTCATC }\end{array}$ \\
\hline ADAM-13 & $\begin{array}{l}\text { Forward PrimerGTGGTGTGGGAAGTATCATCA } \\
\text { Reverse Primer CATCTGGAGTAACCGTATTG }\end{array}$ \\
& Forward PrimerATGACCATGAGGAGCACTACGA \\
Rggrecan & Forward PrimerAGCCTGCGCTCCAATGACT \\
& Reverse Primer GGAACACGATGCCTTTCACC \\
\hline hGAPDH & Forward PrimerGCACCGTCAAGGCTGAGAAC \\
& Reverse Primer TGGTGAAGACGCCAGTGGA \\
\hline
\end{tabular}

\section{Western blot analysis}

Cells were lysed with the precooled RIPA lysate (Solarbio, China). The supernatant was obtained after centrifugation at $4^{\circ} \mathrm{C}$ for $12000 \mathrm{r} / \mathrm{min}$. After adding appropriate amount of sample buffer to the protein sample, the protein samples and standard protein marker were added to polyacrylamide gel and then electrophoretic. After electrophoresis, the protein was transferred to the polyvinylidene fluoride membrane. The membranes immersed in the collagen II antibody (dilution concentration 1:5000; Abcam, China) were incubated overnight in $4^{\circ} \mathrm{C}$. The second antibody (dilution concentration 1:10000; Abcam, China) was added for $1 \mathrm{~h}$. Enhanced chemiluminescence reagent was used to detect the target proteins. $\beta$-Tubulin was used as internal control

\section{Immunofluorescence microscopy}

The cells were cultured in a 24-wells plate. When the cells were covered with coverslips, the chondrocytes were fixed in 4\% PFA (Hyclone, USA) and added with a blocking solution containing $1 \%$ goat serum and $0.5 \%$ Triton $X-100$. The chondrocytes were stained with the primary antibodies of rabbit polyclonal Piezo 1 $\left(1: 400\right.$, Novus, USA) overnight at $4^{\circ} \mathrm{C}$. AlexaFouor $488 \mathrm{Goat}$ Anti-Rabbit IgG was used as secondary antibodies. Then, cell nuclei were stained with $0.1 \mathrm{mg} / \mathrm{mL}$ DAPI (Invitrogen). Images were captured by laser scanning confocal microscopy and analyzed with Image-Pro Plus6.0 software.

\section{Statistical analysis}


The data were processed by SPSS22.0 statistical software, the measurement data were expressed as mean \pm standard deviation (SD), $t$ test were used when the two independent groups of measurement data were compared. One-way ANOVA was used to compare the mean values of multiple samples. $P<0.05$ was defined as statistically significant.

\section{Results}

\section{Culture of the OA chondrocytes}

The OA chondrocytes grew into a polygonal shape (Fig. 1A). Immunohistochemical staining showed that the aggrecan showed a strong positive reaction. The cytoplasm of the chondrocytes was stained with yellow granules, and the nuclear staining was not visible (Fig. 1B).

\section{Transfection of Piezo1-siRNA}

We evaluated the transfection by detecting the expression of green fluorescence protein (Fig. 2A). In addition to the blank control group, the transfection efficiency (fluorescence field of cells / general field of cells $\times 100 \%$ ) of the experimental group was greater than $90 \%$ (Fig. 2B). Compared with experimental group, the transfection efficiency of negative control group had no significant difference. The growth curves of Piezo1-siRNA1-3, blank control, and negative control groups were quite similar (Fig. 2C).

\section{RT-qPCR of Piezo1, MMP-13, ADAMTS5, and Aggrecan}

The mRNA expression characteristics of Piezo1 showed the first increase and then decrease, and reached the maximum expression upon $24 \mathrm{~h}$ mechanical stress (Fig. 3A). Transfected cells were mechanically stress for 24h, qRT-PCR results demonstrated that Piezo1, MMP-13, and ADAMTS5 levels of Piezo1-siRNA2 were reduced compared with Piezo1-siRNA1 and Piezo1-siRNA3, whereas the expression of aggrecan mRNA was presented the opposite trend (Fig. 3B, 3C, 3D, 3E). Piezo1-siRNA2 efficiently inhibits the expression of Piezo1.

\section{Western blotting of Collagen II}

Chondrocytes were mechanically stress for $24 \mathrm{~h}$, and Western blots were used to determine collagen II expression in OA chondrocytes transfected by Piezo1 siRNAs. Compared with the control group, the expression of collagen II in the Piezo1 siRNAs group was significantly increased $(P<0.05)$. Relative collagen expression in siRNA1, siRNA2, and siRNA3 groups was increased approximately $143 \%, 327 \%$ and $212 \%(p<0.01)$ compared to the control group. There was no significant difference in the expression of collagen II between blank control group and negative control group $(P>0.05)$ (Fig. 4).

\section{Immunofluorescence of the Piezo1 in OA chondrocytes}


Cell immunofluorescent analysis revealed that the expression of Piezo1 was detected in OA chondrocytes. Moreover, Piezo1 was primarily located in the nucleus and cell membrane of chondrocytes (Fig. 5).

\section{Discussion}

Piezo1 is a novel type of non-selective mechanically sensitive ion channel which is expressed extensively in mammalian ${ }^{30}$. However, the effect of Piezo1 on cells has not been fully elucidated. The pathogenesis of $\mathrm{OA}$ is related to many risk factors, especially the abnormal mechanical stress has a crucial effect on the progression of $\mathrm{OA}^{31,32}$. Abnormal mechanical stress can not only lead to joint damage in physics but also affect the progress of OA through the baroreceptor signals ${ }^{33}$. Previous studies had reported that TRPV4mediated cartilage mechanotransduction was involved in the pathogenesis of aging- and injury-induced $\mathrm{OA}^{34-36}$. It is also meaningful to explore a novel mechanosensitive ion channel associated with the pathogenesis of OA chondrocytes. Piezo1, a molecule that activates the mechanical signal transduction pathway, is potentially beneficial for the therapy of OA. The excessive destruction of the Extracellular matrix is closely related to the decrease of Chondrocyte ${ }^{37}$. When the synthesis of the extracellular matrix decreases, the living environment of chondrocytes will be destroyed, and it will cause the excessive apoptosis of chondrocytes, which was the critical factor of osteoarthritis ${ }^{38}$. In this research, we tried to investigate the role of Piezo1, a new mechanical sensitive ion channel, in OA chondrocytes. Our results show that Piezo1 acts an effect on the destruction of the extracellular cartilage matrix. The degradation of Collagen II and Aggrecan can be inhibited by siRNA against Piezo1.

The previous study had explored that the presence of extracellular matrix proteins is the prerequisite for Piezo1 to transmit mechanical signals, which discovered that stress patterns could mediate the apoptosis of the cells. However, till now whether Piezo1 acts an effect on the destruction of extracellular matrix is not clear ${ }^{39}$. We speculated that the mechanical stress could activate the Piezo1 and further resulted in the degradation of the Extracellular matrix during the progress of osteoarthritis. In this research, the FAM38A gene encoding Piezo1 was silenced by small interfering RNA, and the chondrocytes stress model was constructed by using a Flexcell Strain Unit. We monitored the expressions of the Piezo1, aggrecan, MMP13, ADAMTS5 with RT-qPCR, and screened the best inhibitory sequence. The expression of Collagen II was detected by Western blot. The expression of the Piezo1, MMP-13, ADAMTS5 were increasing under the mechanical force and had the character of time-dependence, while the col-2 and aggrecan were decreasing. After the FAM38A gene was silenced by Piezo1-siRNA, the expression of the Piezo1, MMP-13, ADAMTS5 were decreasing, while the Collagen II and aggrecan were increasing. The results indicate that Piezo1 had a crucial effect on the degradation of the cartilage matrix and may be served as a potential target for the therapy of $\mathrm{OA}$, especially for those due to trauma.

Understanding the mechanism of the mechanical sensitive ion channel Piezo1 function in chondrocytes is a viable way to understand cartilage mechanical biology and related diseases, especially osteoarthritis. Previous studies have discovered that Piezo1 and TRPV4 are independently activated, while stress induced $\mathrm{Ca}^{2+}$ influx pathway is primarily induced by Piezo1 and TRPV4 channels ${ }^{40}$. There is evidence that L-type 
$\mathrm{Ca}^{2+}$ voltage gated channel can affect $\mathrm{Ca}^{2+}$ influx upon mechanical stress ${ }^{41}$. It is meaningful to speculate that $\mathrm{Ca}^{2+}$ plays an essential role in activating Piezo1.

\section{Conclusions}

In conclusion, the present study found that Piezo1 acts a role on the destruction of the extracellular cartilage matrix. The degradation of col-2 and aggrecan can be inhibited by siRNA against Piezo1. Piezo1, functionally located in OA chondrocytes, provide potential targets for attenuating cartilage matrix degradation and reducing injury-induced cartilage degeneration. In this respect, Piezo1-siRNA could be capable to regard as a cartilage protective agent to prevent and treat osteoarthritis.

\section{Declarations}

\section{Ethical Approval and Consent to Participate}

All methods were carried out in accordance with the Helsinki' s Declaration. All experimental protocols were approved by the medical ethics committee of the Affiliated Hospital of Qingdao University (QYFYKYLL2017-06-12-05), and informed consents were obtained from all patients.

\section{Consent for publication}

All authors consent the publication of this manuscript. Informed consent for publication were obtained from patients involved in the study.

\section{Availability of data and materials}

The datasets generated during and/or analysed during the current study are available from the corresponding author on reasonable request.

\section{Competing interests}

The authors declared no potential conflicts of interest with respect to the research, authorship, and/or publication of this article.

\section{Funding}

This work was supported by the National Natural Science Foundation of China (Grant number 81672197).

\section{Acknowledgment}

Not applicable. 


\section{Authors' Contributions}

All authors participated in the design, interpretation of the studies and analysis of the data and review of the manuscript; Liu Y and Lu X conducted the experiments; Zhang Z and Li T contributed in data curation; Liu Y write the manuscripts; Zhang H: Conceptualization, Supervision, Validation, Writing - review \& editing.

\section{Author details}

${ }^{1}$ Department of Joint Surgery, The Affiliated Hospital of Qingdao University, Qingdao 266000, Shandong, China.

\section{References}

1. Barbour KE, Boring M, Helmick CG, Murphy LB, Qin J. Prevalence of Severe Joint Pain Among Adults with Doctor-Diagnosed Arthritis - United States, 2002-2014. Mmwr-Morbid Mortal W 2016;65:8-12

2. Manek NJ, Lane NE. Osteoarthritis: current concepts in diagnosis and management. Am Fam Physician 2000;61:1795-804

3. Cooper C, Adachi JD, Bardin T, Berenbaum F, Flamion B, Jonsson H, Kanis JA, Pelousse F, Lems WF, Pelletier JP, Martel-Pelletier J, Reiter S, Reginster JY, Rizzoli R, Bruyere O. How to define responders in osteoarthritis. Curr Med Res Opin 2013;29:719-29

4. Oftadeh R, Perez-Viloria M, Villa-Camacho JC, Vaziri A, Nazarian A. Biomechanics and Mechanobiology of Trabecular Bone: A Review. J Biomech Eng-T Asme 2015;137

5. Erdemir A, Bennetts C, Davis S, Reddy A, Sibole S. Multiscale cartilage biomechanics: technical challenges in realizing a high-throughput modelling and simulation workflow. Interface Focus 2015;5

6. Wright T. Biomechanical factors in osteoarthritis: the effects of joint instability. HSS J 2012;8:15-7

7. Smith RL, Trindade MCD, Ikenoue T, Mohtai M, Das P, Carter DR, Goodman SB, Schurman DJ. Effects of shear stress on articular chondrocyte metabolism. Biorheology 2000;37:95-107

8. Chen $\mathrm{MH}$, Broom N. On the ultrastructure of softened cartilage: a possible model for structural transformation. J Anat 1998;192 (Pt 3):329-41

9. Cawston TE, Curry VA, Summers CA, Clark IM, Riley GP, Life PF, Spaull JR, Goldring MB, Koshy PJT, Rowan $A D$, Shingleton WD. The role of oncostatin $M$ in animal and human connective tissue collagen turnover and its localization within the rheumatoid joint. Arthritis Rheum-Us 1998;41:1760-71

10. Lark MW, Bayne EK, Flanagan J, Harper CF, Hoerrner LA, Hutchinson NI, Singer II, Donatelli SA, Weidner JR, Williams HR, Mumford RA, Lohmander LS. Aggrecan degradation in human cartilage - Evidence for both matrix metalloproteinase and aggrecanase activity in normal, osteoarthritic, and rheumatoid joints. J Clin Invest 1997;100:93-106

11. Martel-Pelletier J, Boileau C, Pelletier JP, Roughley PJ. Cartilage in normal and osteoarthritis conditions. Best Pract Res Clin Rheumatol 2008;22:351-84 
12. Julkunen P, Wilson W, Isaksson H, Jurvelin JS, Herzog W, Korhonen RK. A Review of the Combination of Experimental Measurements and Fibril-Reinforced Modeling for Investigation of Articular Cartilage and Chondrocyte Response to Loading. Comput Math Method M 2013;

13. Glasson SS, Askew R, Sheppard B, Carito B, Blanchet T, Ma HL, Flannery CR, Peluso D, Kanki K, Yang ZY, Majumdar MK, Morris EA. Deletion of active ADAMTS5 prevents cartilage degradation in a murine model of osteoarthritis (vol 434, pg 644, 2005). Nature 2007;446:102-02

14. Coste B, Xiao BL, Santos JS, Syeda R, Grandl J, Spencer KS, Kim SE, Schmidt M, Mathur J, Dubin AE, Montal M, Patapoutian A. Piezo proteins are pore-forming subunits of mechanically activated channels. Nature 2012;483:176-U72

15. Li J, Hou B, Tumova S, Muraki K, Bruns A, Ludlow MJ, Sedo A, Hyman AJ, McKeown L, Young RS, Yuldasheva NY, Majeed Y, Wilson LA, Rode B, Bailey MA, Kim HR, Fu ZJ, Carter DAL, Bilton J, Imrie H, Ajuh P, Dear TN, Cubbon RM, Kearney MT, Prasad KR, Evans PC, Ainscough JFX, Beech DJ. Piezo1 Integration of Vascular Architecture with Physiological Force. Faseb J 2015;29

16. Fallahi A, Kroll B, Warner LR, Oxford RJ, Irwin KM, Mercer LM, Shadle SE, Oxford JT. Structural model of the amino propeptide of collagen XI alpha1 chain with similarity to the LNS domains. Protein Sci 2005; 14:1526-37

17. Smith RL, Carter DR, Schurman DJ. Pressure and shear differentially alter human articular chondrocyte metabolism - A review. Clin Orthop Relat R 2004:S89-S95

18. Little CB, Mittaz L, Belluoccio D, Rogerson FM, Campbell IK, Meeker CT, Bateman JF, Pritchard MA, Fosang AJ. ADAMTS-1-knockout mice do not exhibit abnormalities in aggrecan turnover in vitro or in vivo. Arthritis Rheum 2005;52:1461-72

19. Loewenstein WR. Mechanosensitive Channels: Introduction. Biol Bull 1997;192:117

20. Maldonado M, Nam J. The role of changes in extracellular matrix of cartilage in the presence of inflammation on the pathology of osteoarthritis. Biomed Res Int 2013;2013:284873

21. McNulty AL, Leddy HA, Liedtke W, Guilak F. TRPV4 as a therapeutic target for joint diseases. N-S Arch Pharmacol 2015;388:437-50

22. Mocetti P, Silvestrini G, Ballanti P, Patacchioli FR, Di Grezia R, Angelucci L, Bonucci E. Bcl-2 and Bax expression in cartilage and bone cells after high-dose corticosterone treatment in rats. Tissue Cell 2001;33:1-7

23. Mort JS, Flannery CR, Makkerh J, Krupa JC, Lee ER. Use of anti-neoepitope antibodies for the analysis of degradative events in cartilage and the molecular basis for neoepitope specificity. Biochem Soc Symp 2003;70:107-14

24. Gnanasambandam R, Bae C, Gottlieb PA, Sachs F. Ionic Selectivity and Permeation Properties of Human PIEZO1 Channels. Plos One 2015;10

25. Gottlieb PA, Sachs F. Piezo1 Properties of a cation selective mechanical channel. Channels 2012;6:214-19

26. Guilak F. Biomechanical factors in osteoarthritis. Best Pract Res Cl Rh 2011;25:815-23 
27. Griffin TM, Fermor B, Huebner JL, Kraus VB, Rodriguiz RM, Wetsel WC, Cao L, Setton LA, Guilak F. Dietinduced obesity differentially regulates behavioral, biomechanical, and molecular risk factors for osteoarthritis in mice. Arthritis Research \& Therapy 2010;12

28. Gaub BM, Muller DJ. Mechanical Stimulation of Piezo1 Receptors Depends on Extracellular Matrix Proteins and Directionality of Force. Nano Lett 2017;17:2064-72

29. Kim SE, Coste B, Chadha A, Cook B, Patapoutian A. The role of Drosophila Piezo in mechanical nociception. Nature 2012;483:209-12

30. van Tunen JAC, Dell'Isola A, Juhl C, Dekker J, Steultjens M, Lund H. Biomechanical factors associated with the development of tibiofemoral knee osteoarthritis: protocol for a systematic review and metaanalysis. Bmj Open 2016;6

31. Loeser RF. Aging and osteoarthritis: the role of chondrocyte senescence and aging changes in the cartilage matrix. Osteoarthritis Cartilage 2009;17:971-9

32. Kumar D, Manal KT, Rudolph KS. Knee joint loading during gait in healthy controls and individuals with knee osteoarthritis. Osteoarthr Cartilage 2013;21:298-305

33. Chen D, Shen J, Zhao W, Wang T, Han L, Hamilton JL, Im HJ. Osteoarthritis: toward a comprehensive understanding of pathological mechanism. Bone Res 2017;5:16044

34. Wang SP, Chennupati R, Kaur H, Iring A, Wettschureck N, Offermanns S. Endothelial cation channel PIEZO1 controls blood pressure by mediating flow-induced ATP release. J Clin Invest 2016;126:452736

35. Wiren KM, Toombs AR, Semirale AA, Zhang XW. Osteoblast and osteocyte apoptosis associated with androgen action in bone: Requirement of increased Bax/Bcl-2 ratio. Bone 2006;38:637-51

36. Sondergaard BC, Henriksen K, Wulf H, Oestergaard S, Schurigt U, Brauer R, Danielsen I, Christiansen C, Qvist P, Karsdal MA. Relative contribution of matrix metalloprotease and cysteine protease activities to cytokine-stimulated articular cartilage degradation. Osteoarthr Cartilage 2006;14:738-48

37. Sanchez C, Horcajada MN, Scalfo FM, Ameye L, Offord E, Henrotin Y. Carnosol Inhibits ProInflammatory and Catabolic Mediators of Cartilage Breakdown in Human Osteoarthritic Chondrocytes and Mediates Cross-Talk between Subchondral Bone Osteoblasts and Chondrocytes. Plos One 2015;10

38. Hwang HS, Kim HA. Chondrocyte Apoptosis in the Pathogenesis of Osteoarthritis. Int J Mol Sci 2015;16:26035-54

39. Suslak TJ, Watson S, Thompson KJ, Shenton FC, Bewick GS, Armstrong JD, Jarman AP. Piezo Is Essential for Amiloride-Sensitive Stretch-Activated Mechanotransduction in Larval Drosophila Dorsal Bipolar Dendritic Sensory Neurons. Plos One 2015;10

40. Servin-Vences MR, Moroni M, Lewin GR, Poole K. Direct measurement of TRPV4 and PIEZO1 activity reveals multiple mechanotransduction pathways in chondrocytes. Elife 2017;6

41. Yang X, Guan Y, Tian S, Wang Y, Sun K, Chen Q. Mechanical and IL-1beta Responsive miR-365 Contributes to Osteoarthritis Development by Targeting Histone Deacetylase 4. Int J Mol Sci 2016;17:436 


\section{Figures}

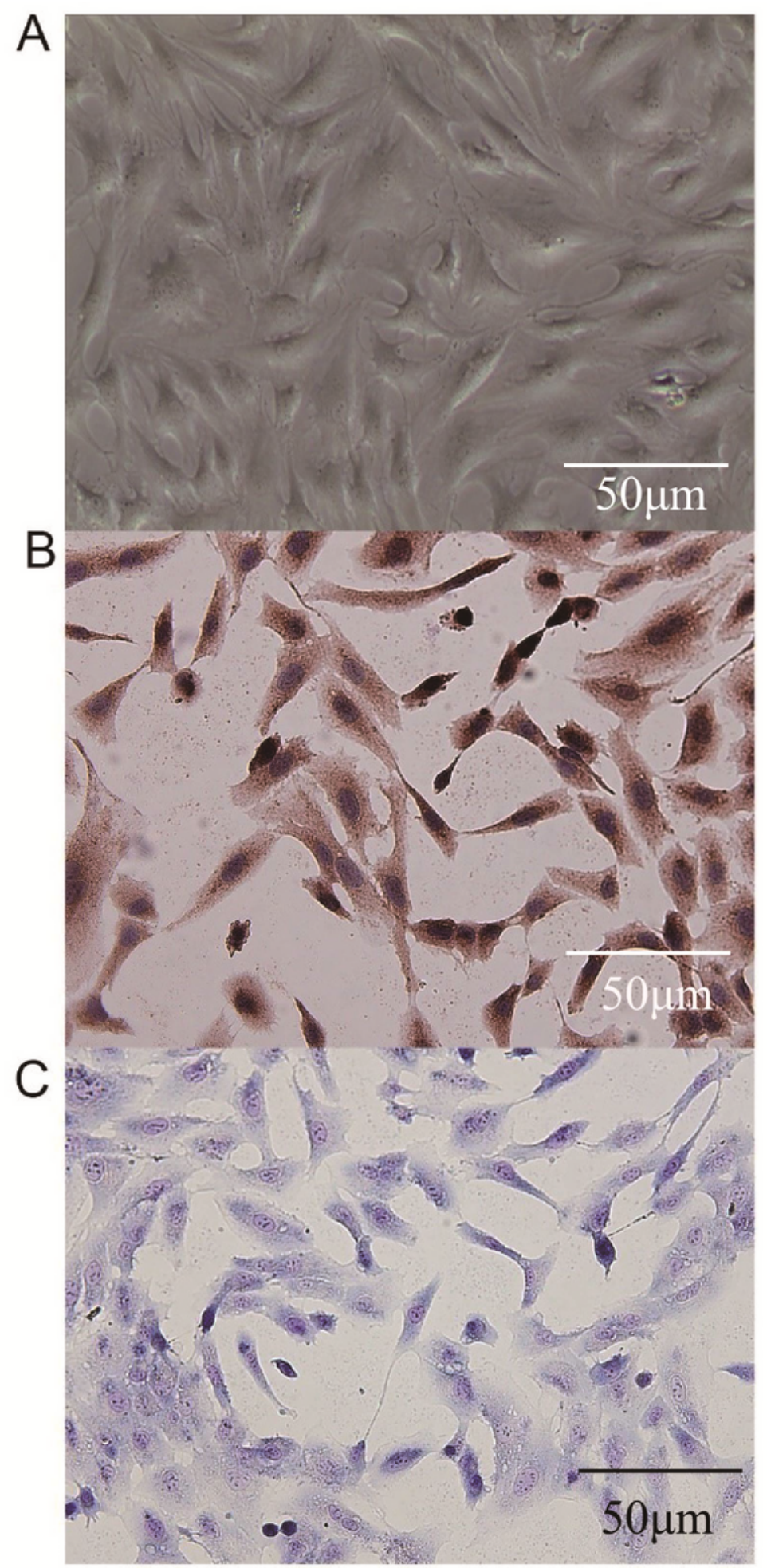

\section{Figure 1}

The OA chondrocytes with Identification and transfection, scale bars, $50 \mu \mathrm{m}$. (A) Primary of OA chondrocytes. (B) OA chondrocytes was also identified by detecting col-2. (C) Toluidine blue staining for primary OA chondrocytes. 

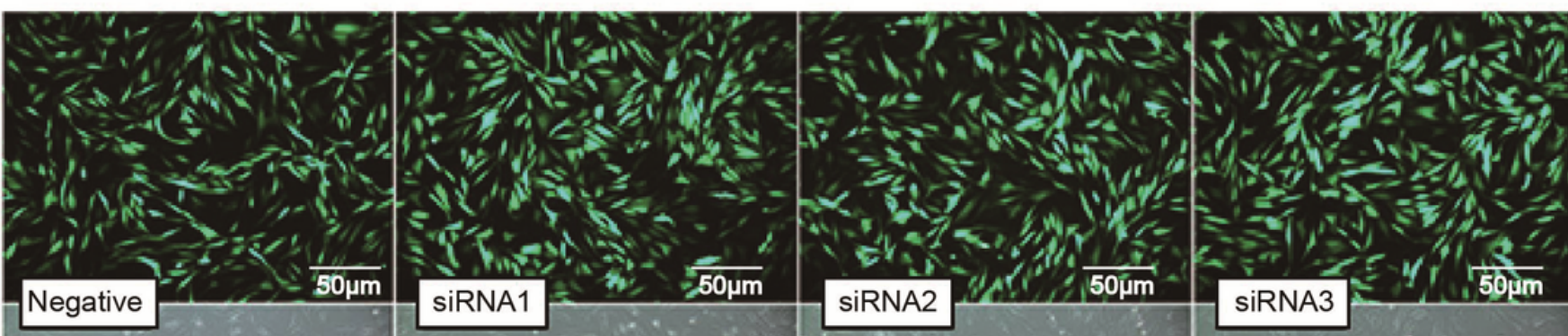

B


Figure 2

The OA chondrocytes with transfection. (A) Green fluorescence protein expression was observed under fluorescence microscope at the end of $48 \mathrm{~h}$ of post-transfection incubation, scale bars, $50 \mu \mathrm{m}$. (B) Fluorescence in each instance was quantified using FACS. The corresponding histogram showed transfection efficiency for Negative random and siRNA1-3. n.s. means P>0.05. (C) Compared with the blank control group, the growth curve of cells in each group was almost parallel. The growth period was from 1 to 2 days, after 3 to 4 days cells turned into the period of growth platform, and then decreased. 

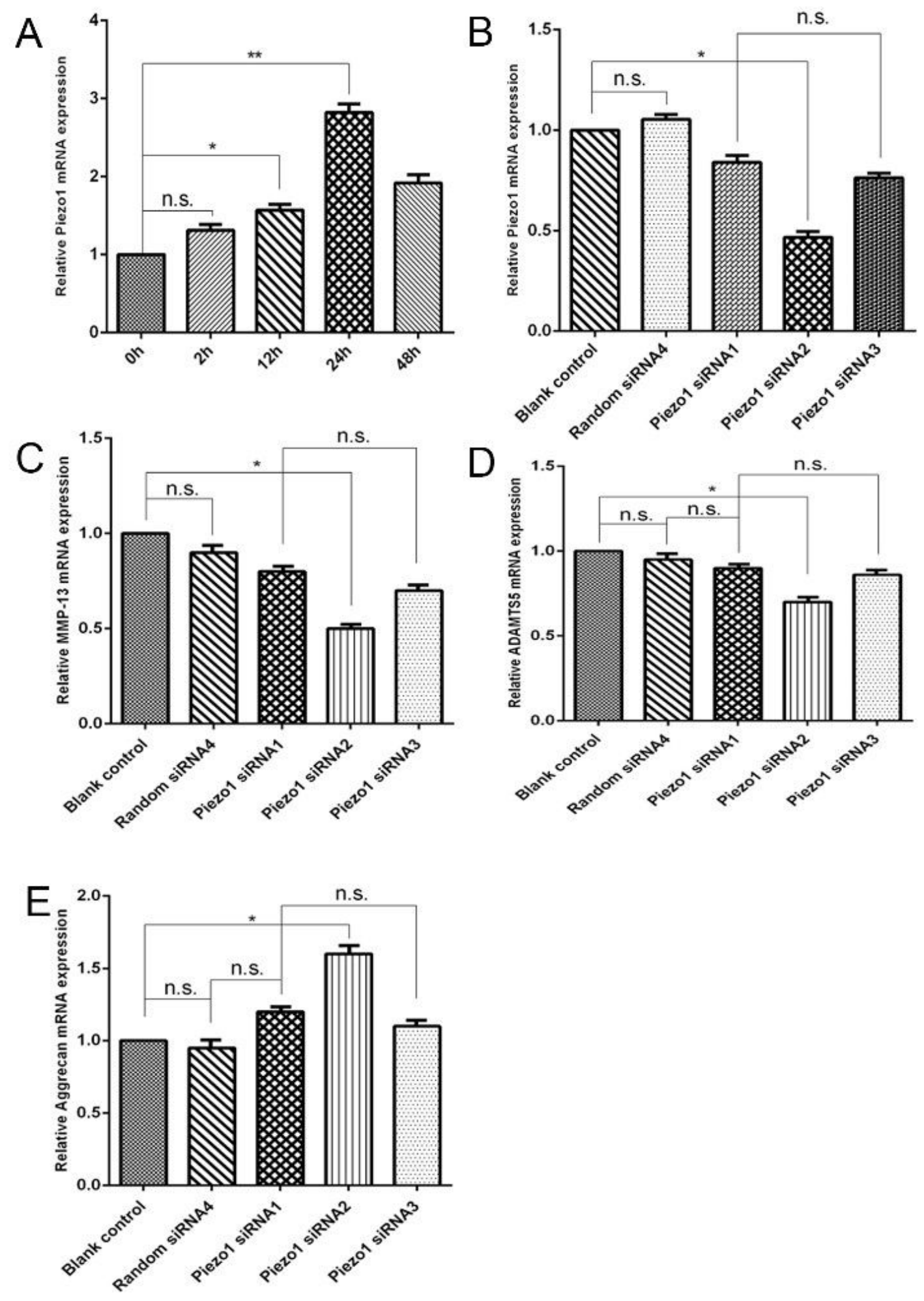

Figure 3

RT-qPCR results of Piezo1, MMP-13, ADAMTS5 and Aggrecan expression in OA chondrocytes. (A) The expression of the Piezo1 in the stress group was increasing under the mechanical stress and had the character of time-dependence. Results represent mean \pm SE. n.s. means $P>0.05$ the mechanical stress group versus the blank group; ${ }^{*}<0.05 * * P<0.01$ the mechanical stress group versus the blank group. $(B, C, D)$ The expression of Piezo1, MMP-13 and ADAMTS5 in small interfering RNA group was decreasing under the 
mechanical stress for 24h. The expression of Piezo1, MMP-13 and ADAMTS5 in siRNA2 group was significantly decreased compared with siRNA1 and siRNA3. (E) Meanwhile, the expression of Aggrecan mRNA was presented the opposite trend.

\section{Collagen II}

\section{$\beta$-tubulin}

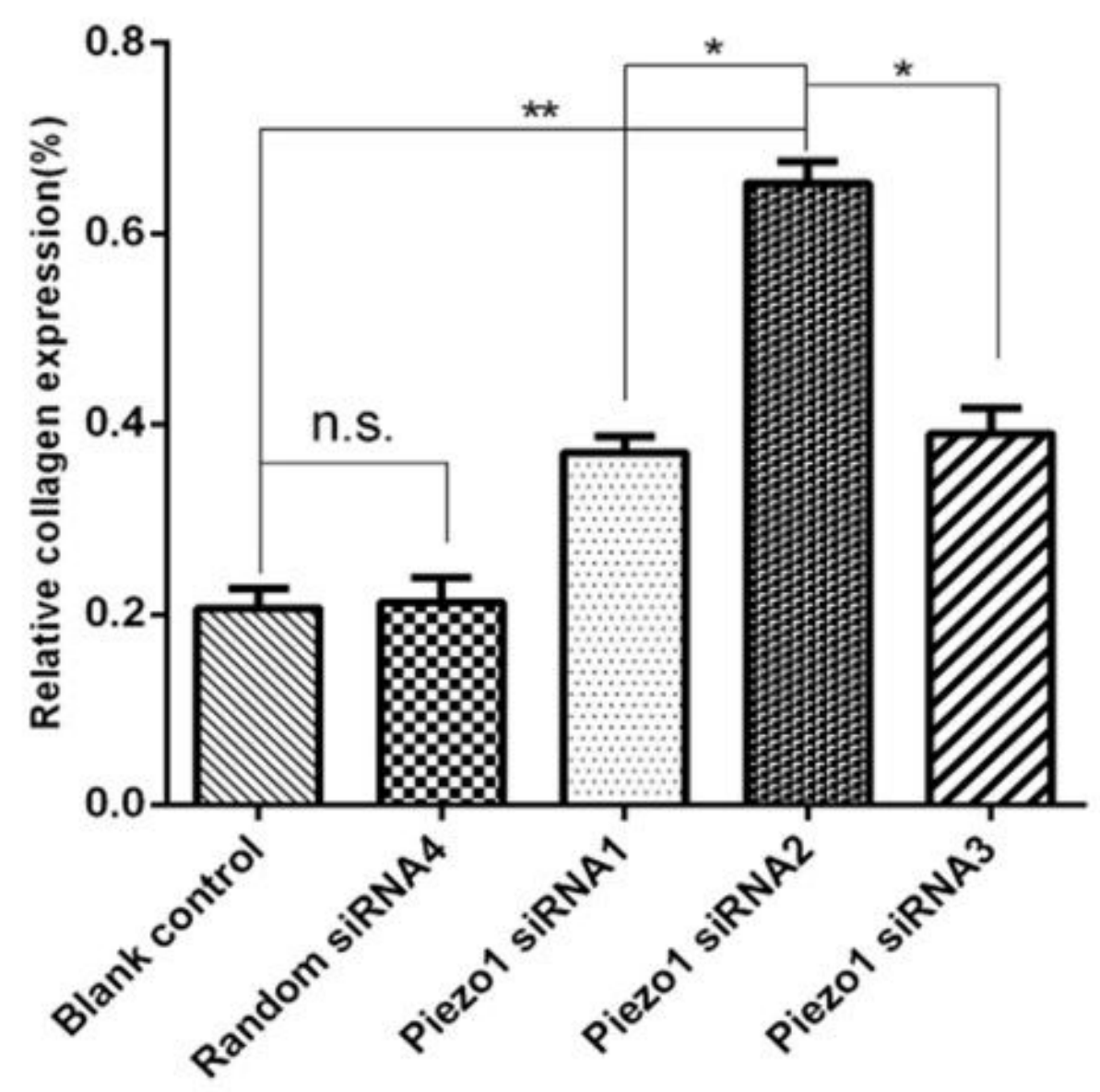

Figure 4

The expression of col-2 regulated by Piezo1-siRNA. The expression level of col-2 in OA chondrocytes was analyzed by western blotting. $\beta$-tubulin was used as internal control. The expression of col- 2 was opposite to that of Piezo1. Under the mechanical stress for $24 \mathrm{~h}, \mathrm{col}-2 \mathrm{in}$ siRNA group was significantly higher than that in control group. In addition, the expression level of col-2 in siRNA2 group was higher than that in siRNA1 and siRNA3 group. Column, mean of three independent experiments; bars, SD; n.s. $P>0.05 * P<0.05$ $* * \mathrm{P}<0.01$. 




\section{Figure 5}

LSCM results of the Piezo1 protein-specific immunolabeling in the OA, scale bars, $5 \mu \mathrm{m}$.

\section{Supplementary Files}

This is a list of supplementary files associated with this preprint. Click to download.

- GraphicalAbstractlmage.jpg

- GraphicalAbstractText.docx 